\title{
Real-time Video Quality Assessment for Analog Television Based on Adaptive Fuzzy Membership Function Tuning
}

\author{
Herti Miawarni ${ }^{*}{ }^{1}$, M. Mahaputra Hidayat ${ }^{2}$, Surya Sumpeno ${ }^{3}$, Eko Setijadi ${ }^{4}$ \\ ${ }^{1,2}$ Engineering Faculty, Bhayangkara Surabaya University, Indonesia \\ ${ }^{3,4}$ Faculty of Electrical Technology, Institut Teknologi Sepuluh Nopember Surabaya, Indonesia \\ *Corresponding author, e-mail: herti_mia@ubhara.ac.id
}

\begin{abstract}
Real-time VQA (Video Quality Assessment) is an important part in the effort to build tracking antenna system especially for analog TV. In this case, VQA must work in real-time to assess the video clarity level. VQA assessment results are valuable information for the decision-making process. Thus, the antenna can rotate automatically looking for the ideal direction without user's control. In addition, the video clarity level on the TV screen can reach optimum according to the user's wishes. The biggest challenge to VQA is, VQA must be able to assess the video clarity level according to the user's visual perception. Therefore, in this study, the MOS-VQS (Mean Opinion Score-Video Quality Subjective) was used as a visual perception approach. In addition, Adaptive FIS (Fuzzy Inference System) with membership function tuning was implemented for decision making. This was conducted as an effort to build a reliable real-time VQA. The test results show that real-time VQA that has been built has a good performance. This is shown from the average accuracy percentage of the lowest assessment reached $77.2 \%$ and the highest reached $88.2 \%$.
\end{abstract}

Keywords: Real-time VQA, adaptive FIS, membership function tuning, tracking antenna system, analog TV

Copyright $\odot 2018$ Universitas Ahmad Dahlan. All rights reserved.

\section{Introduction}

On analog TV, video clarity level is the video parameter that determines QoE (Quality of Experience). The video clarity level on analog TV is not only influenced by RF strength, but also influenced by antenna direction. Tracking antenna system is technology that required so that TV antenna direction is in the ideal states automatically. We assume that the study on tracking antenna systems for analog TV is very important because of the fact that TV antennas with auto tracking capabilities are not popular on the market. Auto tracking capabilities on tracking antenna systems allow the antenna to rotate automatically and understand the video clarity level on the user's TV screen. So, the main problem is how to realize reliable tracking antenna system that has auto tracking capabilities for analog TV reception. We assume that there are 4 points that are needed to build the tracking antenna system, among others are:

a. Input parameters that used as reference for the tracking antenna system.

b. VQA (Video Quality Assessment) that works in real time to assess video quality, especially video clarity level. VQA should have reliable algorithm to video quality assessing, and also a visual perception approaches. This is because the visual perception on the built system must approximate the user's visual perception in assessing video quality.

c. Final decision making that determining antenna direction, tracking direction, tracking time, idle time and, etc.

d. Antenna rotator construction and antenna design.

The general purpose of this study is to produce the applicable technology for analog TV reception. In particular, to build a reliable tracking antenna system. In this case, the reliability of the tracking antenna system depends on real-time VQA reliability. While the specific purpose of this study is about how to build reliable real-time VQA (point $b$ ) that include algorithm for VQA and visual perception approaches. But in this case, real time VQA cannot stand alone without 
any input parameter (point a). So, in this study, parameter input, algorithm for VQA and visual perception approaches will be discussed.

First discussion is about input parameter. Tracking antenna systems require information about the video clarity level on the user's TV screen. This information is obtained from the AV-Out (Audio Video Output) connection on the rear panel of the TV. This connection produces CVBS signals continuously. The CVBS (Composite Video Baseband Signal) on AV-Out can describe the video clarity level [1]. Tracking antenna system based on CVBS signal processing has been proposed in previous studies [2]. However, both of previous study has not been developed in terms of VQA and visual perception.

Second discussion is about algorithm for VQA, Assessment of the video clarity level is one form of the decision-making process. To achieve optimal results, the decision-making process should be simple, easy to control, and easy to interact with users. One of the most common algorithms used in the decision-making process is FIS (Fuzzy Inference System). In the previous study, FIS has been used for decision-making in an effort to improve the quality of information [3]. The implementation of FIS (Fuzzy Inference System) on VQA has been widely used, fuzzy rule evaluation has been proposed to measure image quality due to distortion based on HVS (Human Visual System) modeling [4]. FIS also used to predict the quality of video from several fidelity distortions, blurring distortion and contrast distortion [5]. FIS has been proposed to improve QoE (Quality of Experience) by building quality predictions on mobile device-based video [6-7]. In addition, FIS has also been used to predict the quality of wireless video streaming [8-9]. While in current study, FIS used as VQA for the tracking antenna systems that work in real-time and make faster and precise decision-making related to assessing video clarity level.

Third discussion is about visual perception approaches. On video quality measurement, it is necessary to understand how users generally assess a video and its quality. So, visual perception is complex [10]. The popular method used to calculate video quality is PSNR (Peak Signal-to-Noise Ratio) or by using MSE (Mean Square Error) [11-14]. Another method that also often used is MOS (Mean Opinion Score) approaching. Previous study related to video quality measurement in analog TV technology is using MOS approach to video quality with random noise. The parameters used are the comparison of chrominance and luminance signal versus noise [15]. While in this study, the MOS approach uses another parameter that is the result of CVBS signal processing. MOS variant that used is MOS-VQS (Mean Opinion Score-Video Quality Subjective) because video clarity level assessment is subjective and it's limited to noise and ghosting influence on analog video TV quality.

Considering to previous discussion, the real-time VQA in this study was built from FIS algorithm for decision making process in assessing video clarity, MOS-VQS for visually perceptual approach and CVBS signal processing result for VQA's input parameters. Besides that, the membership function on FIS can be changed according to user's perception. This is conducted so that the real time VQA built can adaptive to many diverse users visual perception. So, the FIS algorithm that used in this study is adaptive FIS with membership function tuning.

\section{Methodology}

In an effort to realize reliable real-time VQA, the research methodology steps begin with the system design that includes hardware design and algorithm design. Then the second step is FIS algorithm simulation that includes membership function tuning. The third stage is real-time VQA performance test that includes VQA performance trial on default mode and VQA performance trial on user mode. User mode and default mode will be discussed on sub-chapter 3.4. The final step is overall results analysis. The research methodology is as shown in Figure 1.

\section{System Design}

Figure 2 shows the system design of real-time VQA that proposed. FIS algorithm that serve as assessment machine are designed with visual perception approaches using MOS-VQS. The input of real-time VQA is the result of CVBS signal processing. The real-time VQA output is accurate information with regard to the video clarity level on the user's TV screen. 
Due to the focus of this study is limited to real-time VQA, video clarity level information that produce by real-time VQA will be displayed on the LCD display and then recorded as trial result.

Each user certainly has a different perception in assessing the video clarity level. Therefore, this study proposed an access control for users. This access control is connected to the fuzzification process on the FIS. It is expected that the real-time VQA performance can correspond with the user's desires.

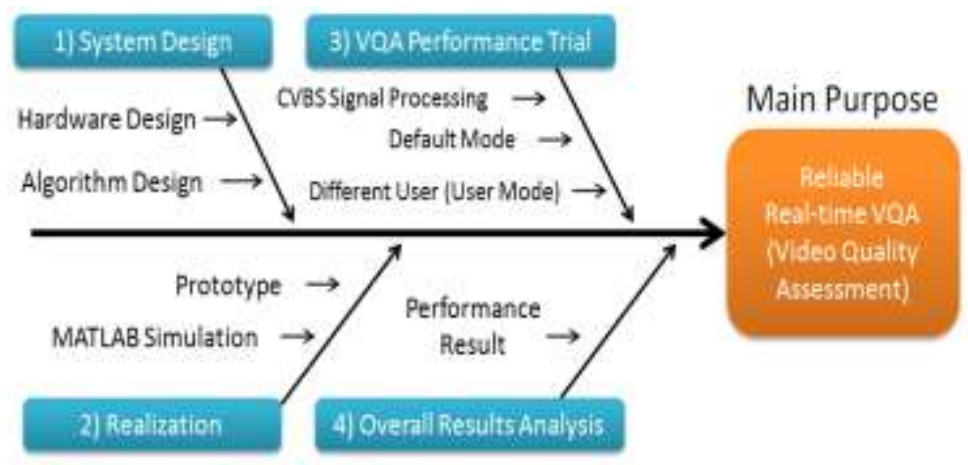

Figure 1. Research Methodology

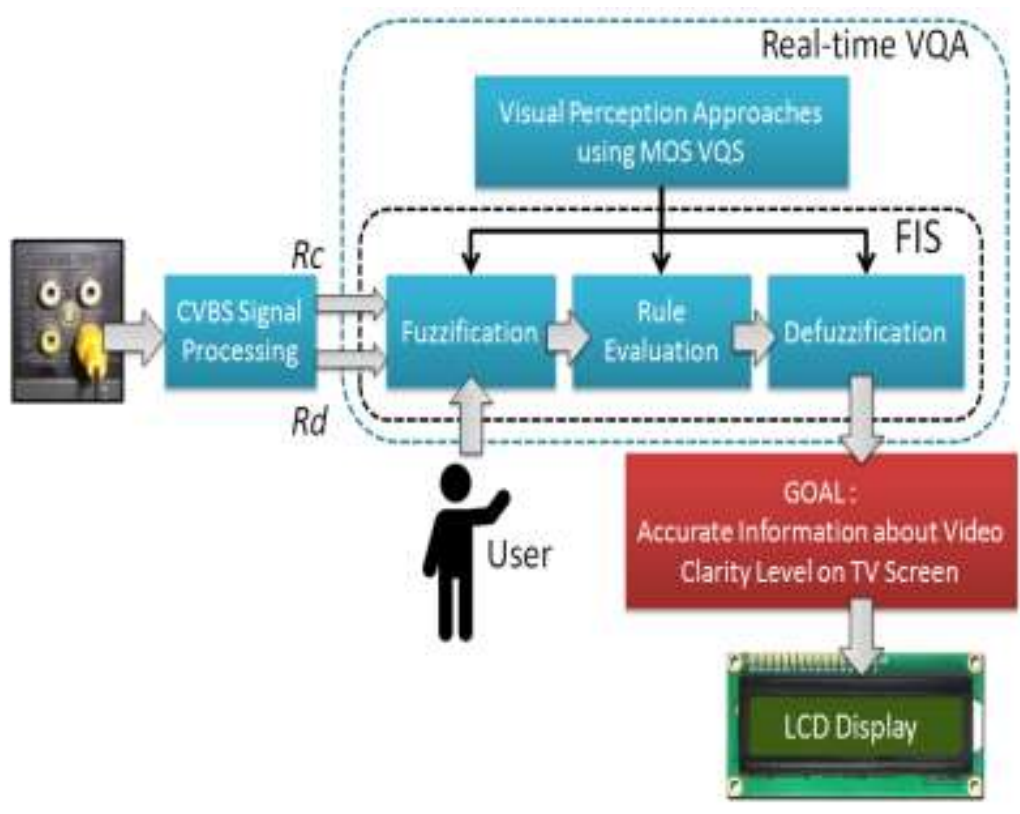

Figure 2. System Design of Real-time VQA

\subsection{Visual perception approaches using MOS-VQS}

MOS-VQS (Mean Opinion Score-Video Quality Subjective) is a subjectively visual perception approaches and this is a recommendation from ITU-T (International Telecommunication Union-Telecommunication standard section). Most of the MOS-VQS use 5 points quality scaling, among others are excelent, good, fair, poor, and bad [16]. But in this study, 5 scale were initialized as (good clarity, fair clarity, bad clarity, worst clarity, noisy). Then, each scale is visualized to the TV reception characteristics in the urban area as in the Table 1. Picture in the Table 1 obtained from observation on latitude and longitude $7^{\circ} 19^{\prime} 12.0 \mathrm{~S} 112^{\circ}$ 43'55.9_E. This coordinate is an area with many tall buildings that can disturb TV signal reception. 
Table 1. Subjectively Video Clarity Level Classification with MOS-VQS [2]

\begin{tabular}{|c|c|c|c|}
\hline MOS Scale Standard & $\begin{array}{l}\text { Scale of Video Clarity Level } \\
\text { in This Study }\end{array}$ & $\begin{array}{l}\text { TV reception } \\
\text { characteristics }\end{array}$ & Description \\
\hline 1. Excellent & Good Clarity & & $\begin{array}{l}\text { Perfect colors and } \\
\text { minimum ghosting. }\end{array}$ \\
\hline 2. Good & Fairly Clarity & & $\begin{array}{l}\text { Perfect colors but } \\
\text { fairly ghosting. }\end{array}$ \\
\hline 3. Fair & Bad Clarity & & $\begin{array}{l}\text { Blurred colors and } \\
\text { ghosting looks dominant. }\end{array}$ \\
\hline 4. Bad & Worst Clarity & & $\begin{array}{l}\text { Ghosting looks } \\
\text { extremely dominant. }\end{array}$ \\
\hline 5. Worst & Noisy & & $\begin{array}{l}\text { Noise looks extremely } \\
\text { dominant, } \\
\text { image and colors are } \\
\text { disappear. }\end{array}$ \\
\hline
\end{tabular}

\subsection{CVBS signal processing}

Tracking antenna system requires the input parameter as a reference. The AV-Out connection on the rear panel of the TV is the most potential item to used. The signal format on AV-Out is a CVBS analog signal. As in previous studies [1]-[2], counting the synchronization pulse on a CVBS signal is one way of obtaining information related to the video clarity level. The reason for using this way is because the synchronization pulse of CVBS signal on AV-Out depends on the video clarity level on the TV screen. If video clarity level on TV screen is becomes better, then shape and number of synchronization pulse on AV-Out becomes close to the CVBS standard. Figure 3 shows the design of CVBS signal processing.

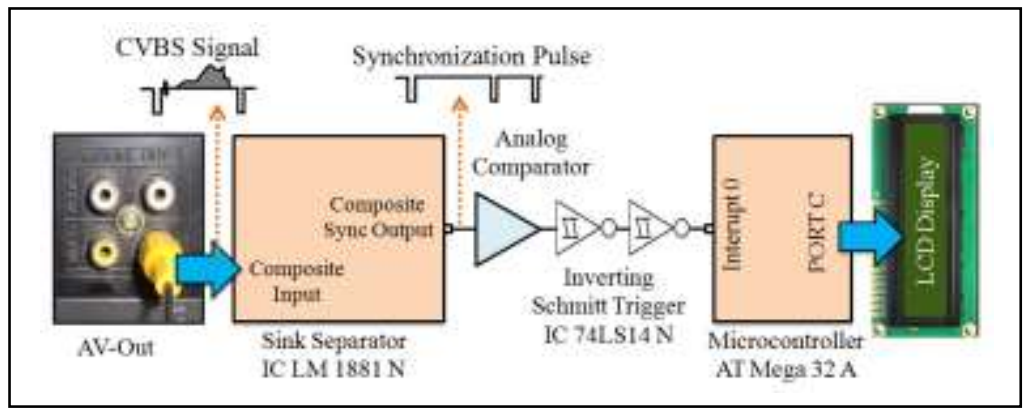

Figure 3. Design of CVBS Signal Processing [2]

To calculate the synchronization pulse, a sync separator IC LM $1881 \mathrm{~N}$ used to separate the synchronization pulse from other signals (luminance signals, chrominance signal and burst signal). Analog comparator and inverting Schmitt trigger is used to ensure that voltage level of synchronization pulse is sufficient and can be processed by microcontroller. Then, synchronization pulse calculations are performed by timer counter on the AT-Mega 
32 microcontroller. In this case, the microcontroller calculates the rising edge of the transition between sync level and blanking level on the CVBS signal. In this study, the results of calculations called with rising transition and it will be processed using FIS algorithm. In this case, FIS used as real-time VQA. Real-time VQA output will produce values that describe the video clarity level accurately. Then the information will be displayed on the LCD display. In this study, FIS algorithm implemented into listing program on AT-Mega microcontroller $32 \mathrm{~A}$.

PAL BG (Phase Alternating by Line, $B$ and $G$ variant) is color encoding standard that used for analog television in Indonesia. In PAL BG, each frame contains 625 lines which divided into 2 fields (odd and even) [17]. The number of rising transition on the overall synchronization pulse are 640 per frame. This value is derived from the summing transition of each line. If every second there are 25 frames, then the rising transition number are 16,000 per second. The rising transition on CVBS signal sourced from AV-Out has special characteristics. The number of rising transition will be closer to 16,000 if the video clarity is better, and vice versa. In the previous study, rising transition used as the main parameter [1]. As a continuity of previous study, then in this study conducted development. In this study used two parameters which in this case we call as rising transition difference $(R d)$ and rising transition changes $(R c)$. Details of both parameters are as follows :

a. Rising transition difference $(R d)$ is the difference between rising transition counting results with the standard value of 16,000 . The smaller the value of this parameter, the video clarity level condition can be defined as becomes better and vice versa. Rising transition difference $(R d)$ is a single integer variable which is updated in real-time every second. Rising transition difference (Rd) can be expressed in equation (1).

$$
R d=A b s[16,000-\text { rising transition }]
$$

b. Rising transition changes $(\mathrm{Rc})$ is the difference between the current rising transition difference $\left(R d_{(\text {Now) }}\right)$ with the previous rising transition difference $\left(R d_{(\text {Previous })}\right)$. The smaller the value of this parameter, the video clarity level condition can be defined as becomes better and vice versa. Rising transition changes (Rc) is an integer and is not a negative value. Rising transition changes (Rc) can be expressed in equation (2).

$$
R c=A b s\left[R d_{(\text {now })}-R d_{(\text {Previous })}\right]
$$

\subsection{FIS for assessment machine}

\subsubsection{Zero order fuzzy sugeno}

In this study, zero order fuzzy sugeno used as real-time VQA. The reason for using zero order is a lightweight computational load with the assumption that the microcontroller as the main processor has limited computing capability. Input of FIS obtained from two parameters, namely, rising transition difference (Rd) and rising transition changes (Rc). While the output parameters of the FIS are information related to the video clarity level. In the fuzzification process, the design of membership function on the input parameters in this study is arranged as in Figure 4. While the designing membership function on the output parameters shown in Figure 5.
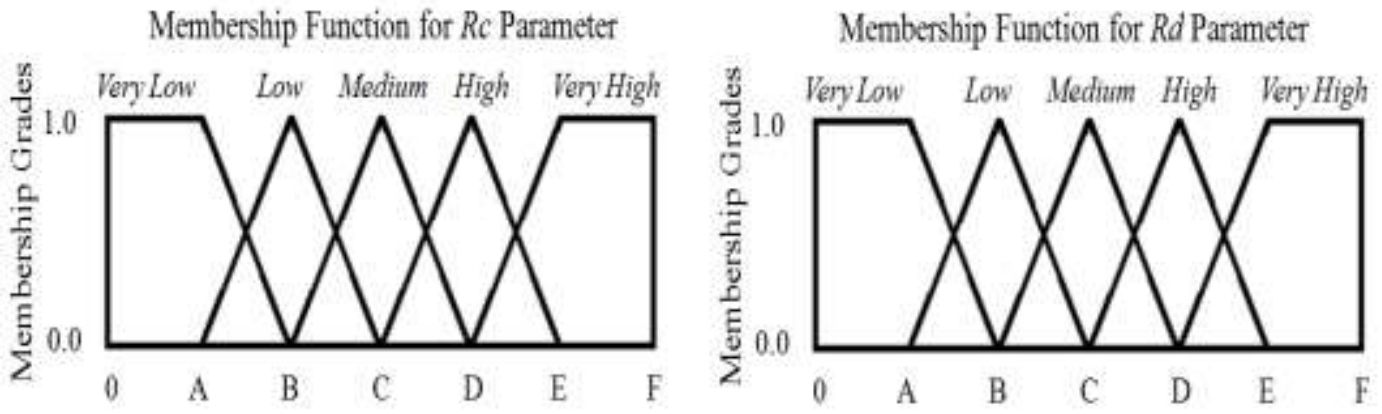

Figure 4. Membership Function for $R d$ and $R c$ Parameter 


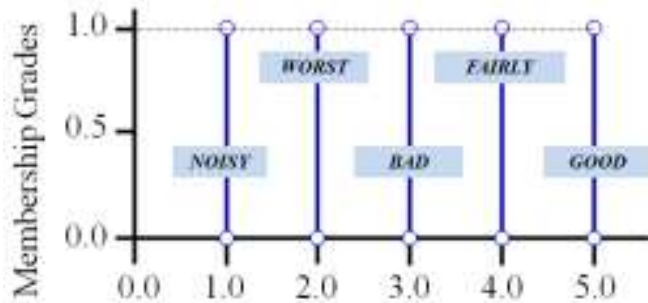

Information About Video Clarity Level

Figure 5. Membership Function for Output Parameter

After the fuzzification process, then the next process is to determine the fuzzy rule. As many as 25 rules used in the fuzzy rule which shown as detail in Table 2. In the defuzzification process, the Weighted Average method used because it has relatively lightweight computing load [18]. Furthermore, the defuzzification process is expressed in the equation (3).

$$
Z=\frac{\sum \mu C(Z n) \cdot Z n}{\sum \mu C(Z n)}
$$

Where:

a. $z$ is the output value sought. This value is real (float) number that represents the video clarity level. Furthermore, this value will be replaced with information by MOS-VQS scale, among others are: $G O O D$ (for $4.6 \geq z \leq 5$ ), FAIRLY (for $3.6 \geq z \leq 4.5$ ), $B A D$ (for $2.6 \geq z \leq 3.5$ ), WORST (for $1.6 \geq z \leq 2.5$ ) and NOISY (for $1 \geq z \leq 1.5$ ).

b. $\mu C$ is minimum value of membership grades on $R d$ and $R c$ parameters. For in this case, $\mu C>0 \leq 1$.

c. $Z n\left[Z_{1} \ldots Z_{25}\right]$ value of fuzzy rule number to- $n\left(Z_{n}\right)$ which is not zero. For in this case, $1 \geq Z_{n} \leq 5$.

Table 2. Fuzzy Rule

\begin{tabular}{clll}
\hline $\begin{array}{c}\text { Fuzzy Rule } \\
\text { Number }\end{array}$ & \multicolumn{1}{c}{$\begin{array}{c}\text { Rising Transition } \\
\text { Difference }\end{array}$} & \multicolumn{1}{c}{$\begin{array}{c}\text { Rising Transition } \\
\text { Changes }\end{array}$} & \multicolumn{1}{c}{$\begin{array}{c}\text { Video Clarity Level Information Displayed } \\
\text { on LCD Display }\end{array}$} \\
\hline $1\left(\mathrm{Z}_{1}\right)$ & very low & very low & GOOD $(5.0)$ \\
$2\left(\mathrm{Z}_{2}\right)$ & very low & low & GOOD $(5.0)$ \\
$3\left(\mathrm{Z}_{3}\right)$ & very low & medium & FAIRLY $(4.0)$ \\
$4\left(\mathrm{Z}_{4}\right)$ & very low & high & BAD $(3.0)$ \\
$5\left(\mathrm{Z}_{5}\right)$ & very low & very high & WORST $(2.0)$ \\
$6\left(\mathrm{Z}_{6}\right)$ & low & very low & WORST $(5.0)$ \\
$7\left(\mathrm{Z}_{7}\right)$ & low & low & FAIRLY $(4.0)$ \\
$8\left(\mathrm{Z}_{8}\right)$ & low & medium & FAIRLY $(4.0)$ \\
$9\left(\mathrm{Z}_{9}\right)$ & low & high & BAD $(3.0)$ \\
$10\left(\mathrm{Z}_{10}\right)$ & low & very high & WORST $(2.0)$ \\
$11\left(\mathrm{Z}_{11}\right)$ & medium & very low & FAIRLY $(4.0)$ \\
$12\left(\mathrm{Z}_{12}\right)$ & medium & low & FAIRLY $(4.0)$ \\
$13\left(\mathrm{Z}_{13}\right)$ & medium & medium & BAD $(3.0)$ \\
$14\left(\mathrm{Z}_{14}\right)$ & medium & high & BAD $(3.0)$ \\
$15\left(\mathrm{Z}_{15}\right)$ & medium & very high & WORST $(2.0)$ \\
$16\left(\mathrm{Z}_{16}\right)$ & high & very low & BAD $(3.0)$ \\
$17\left(\mathrm{Z}_{17}\right)$ & high & low & BAD $(3.0)$ \\
$18\left(\mathrm{Z}_{18}\right)$ & high & medium & BAD $(3.0)$ \\
$19\left(\mathrm{Z}_{19}\right)$ & high & high & WORST $(2.0)$ \\
$20\left(\mathrm{Z}_{20}\right)$ & high & very high & NOISY $(1.0)$ \\
$21\left(\mathrm{Z}_{21}\right)$ & very high & very low & NOISY $(1.0)$ \\
$22\left(\mathrm{Z}_{22}\right)$ & very high & low & NOISY $(1.0)$ \\
$23\left(\mathrm{Z}_{23}\right)$ & very high & medium & NOISY $(1.0)$ \\
$24\left(\mathrm{Z}_{24}\right)$ & very high & high & NOISY $(1.0)$ \\
$25\left(\mathrm{Z}_{25}\right)$ & very high & very high & \\
\hline & & &
\end{tabular}




\subsubsection{Diversity of users satisfaction and adaptive fis with membership function tuning}

In this study, each user's is assumed to have different levels of satisfaction against video clarity level on the TV screen. The diversity of user satisfaction levels will cause diversity of visual perceptions. On the other hand, the level of satisfaction also affects the tracking time needed for the tracking antenna system. In this case, not all users want to watch TV if video clarity level on the TV screen is only on good clarity condition and causes long tracking time.

In order for real-time VQA to match with the user's visual perceptions diversity, real-time VQA is designed to be able to receive manual input from the user. Thus, real-time VQA performance can be closer to the user satisfaction level. Therefore, in this research, adaptive FIS with membership function tuning is proposed and implemented. Membership function does not change automatically, but it can change flexibly to the user's perception.

In realization, users are given access to change membership function by simply pressing a button. It is intended to users can convey the visual perception to the system directly and easily without having to understand what is FIS algorithm. Users only need to press the GOOD button when the video clarity level is in good clarity condition, pressing the FAIRLY button when the video clarity level is in fairly clarity condition, and so on. Then the FIS algorithm will work in real-time to assess the video clarity level and display the information on the LCD. Then this information used for decision-making process on real-time VQA. Figure 6 shows the realization of real-time VQA. Referring to Figure 4 and Figure 6, the working principle of FIS with membership function tuning is as follows:

a. If user presses the GOOD button as shown in Figure 6 then microcontroller will extract the $\mathrm{Rd}$ and Rc parameters values which obtained by CVBS signal processing. Then store the value into variable $A$ as shown in Figure 4 on fuzzy membership function.

b. If user presses the FAIRLY button as shown in Figure 6 then microcontroller will extract the $\mathrm{Rd}$ and Rc parameters values which obtained by CVBS signal processing. Then store the value into variable $B$ as shown in Figure 4 on fuzzy membership function.

c. If user presses the BAD button as shown in Figure 6 then microcontroller will extract the Rd and Rc parameters values which obtained by CVBS signal processing. Then store the value into variable $C$ as shown in Figure 4 on fuzzy membership function.

d. If user presses the WORST button (see Figure 6) then microcontroller will extract the Rd and Rc parameters values which obtained by CVBS signal processing. Then store the value into variable $D$ as shown in Figure 4 on fuzzy membership function.

e. If user presses the NOISY button (see Figure 6) then microcontroller will extract the Rd and Rc parameters values which obtained by CVBS signal processing. Then store the value into variable $E$ as shown in Figure 4 on fuzzy membership function.

f. After pressing the button, A, B, C, D and E, the value become differ for each video clarity level (good clarity, fair clarity, bad clarity, worst clarity, and noisy). Besides that, A, B, C, D and $E$ value also become differ for each user's subjectivity. It will produces the triangular legs in membership function has different sides and changeable according to user's perception.

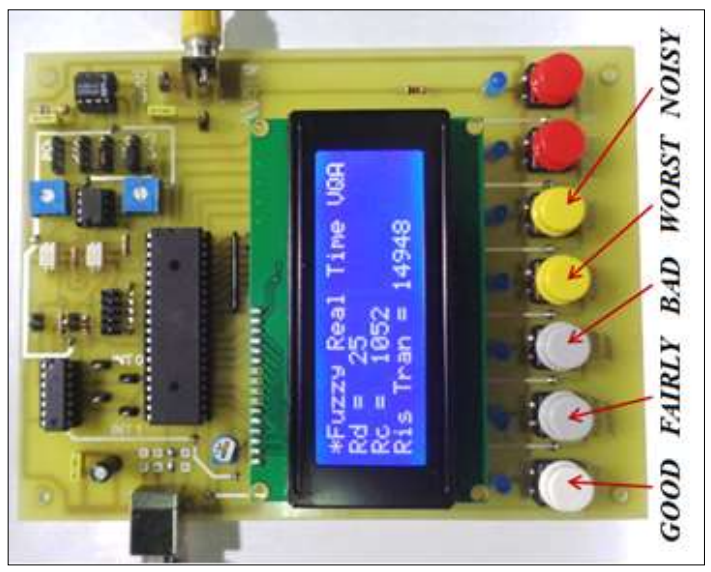

Figure 6. Realization of Real-time VQA 


\subsection{Trial scenario (user mode and default mode)}

On the implementation, the assessment on real-time VQA is obtained from user's assessment (User Mode) and from default assessment (Default Mode). In User Mode, the membership function of FIS algorithm is determined by user. As shown in Figure 6, the PCB prototype provides a button for the user to enter input assessment which indirectly gives access to change the membership function settings on the FIS algorithm. While in Default Mode, membership functions in the FIS algorithm follow the listing program that has been compiled at early time. Membership function is determined based on CVBS signal processing measurement results and after MATLAB simulation process.

\section{Results and Analysis}

\subsection{CVBS signal processing characteristic}

The trial was conducted to observe the values of rising transition difference $(R d)$ and rising transition changes $(\mathrm{Rc})$ parameters in some video clarity conditions. The results of this trial, then will be used as a reference in preparing the membership function FIS algorithm, especially in Default Mode. The result of the trial in this session is as shown in Figure 7 and Figure 8.

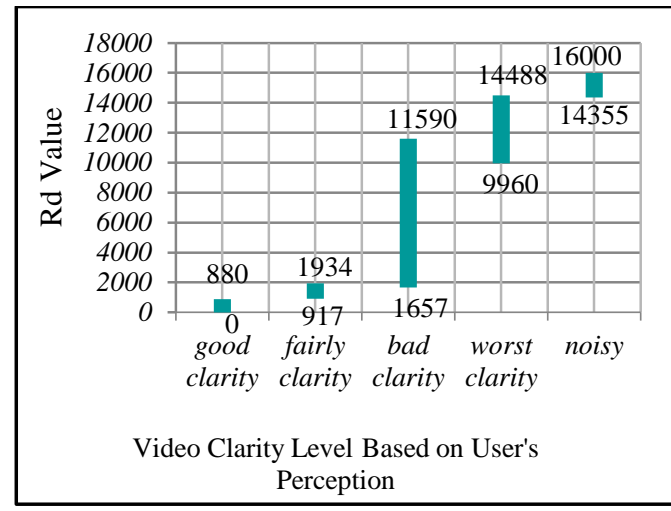

Figure 7. Rd Parameters at Various Video Clarity level

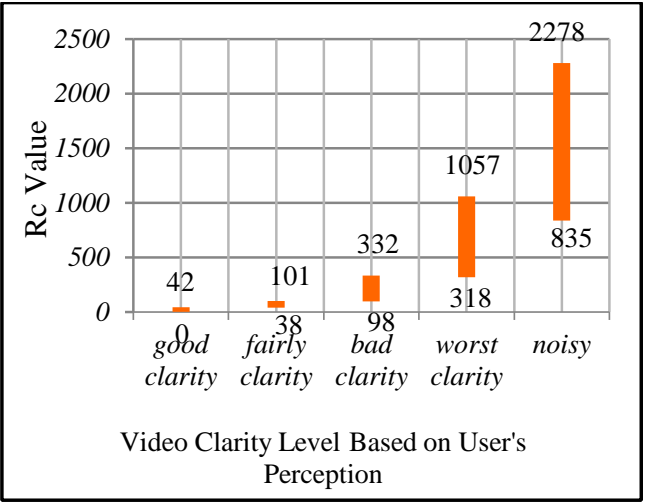

Figure 8. Rc Parameters at Various Video Clarity Level

The test result in Figure 7 shows that the value of the Rd parameter in a good clarity condition is smaller when compared to the noisy state. In good clarity condition, the minimum value is 0 and the maximum reaches 880 . While in the noisy condition, the minimum value reaches 14,355 and the maximum reaches 16,000 . The widest range of values is obtained from bad clarity conditions. In this condition, the value of the Rd parameter is in the range of 1,657 to 11,590 . Overall, it can be concluded that, if video clarity level becomes better, and then value of the Rd parameter becomes smaller. Conversely, if video clarity level becomes worse, then value of the Rd parameter becomes greater.

The test result in Figure 8 shows that the value of the Rc parameter in a good clarity condition is smaller when compared to the noisy condition. In good clarity condition, the minimum value is 0 and the maximum reaches 42 . While in the noisy condition, the minimum value reaches 835 and the maximum reaches 2,278. Overall, it can be concluded that, the better the video clarity, the smaller the Rc value changes in every second. In this case, the value of Rc can be said to be more stable when compared with other conditions. Conversely, if the video clarity becomes worse, then value of the Rc parameter will become unstable.

The test results in Figure 7 and Figure 8 show the difference in values of Rd and Rc parameters at some video clarity level. In other words, the Rd and Rc parameters are feasible to be used as fuzzy input parameters in an effort to build a reliable real-time VQA. Then, using the test results, the membership function in Default Mode is designed as in Figure 9. In this case, the membership function is triangular with different sides, because it adjusts the range of Rd 
and Rc parameter values according to the test results in Figure 7 and Figure 8 . While Figure 10 shows the rule surface of the simulation result using MATLAB toolbox.

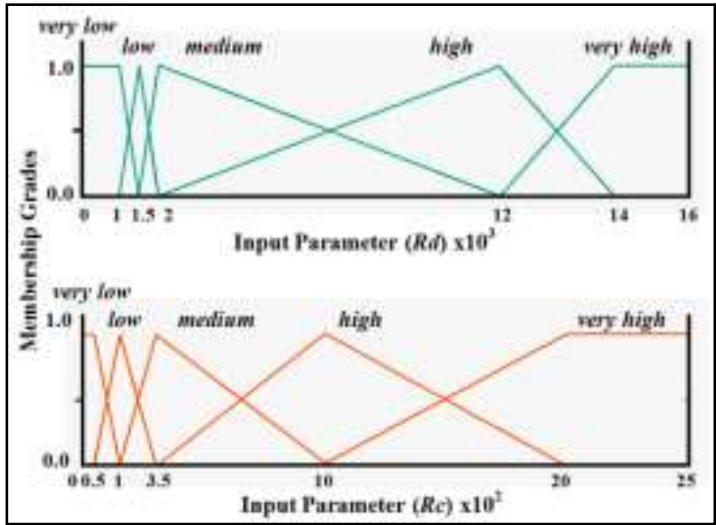

Figure 9. Membership Function Design on Default Mode

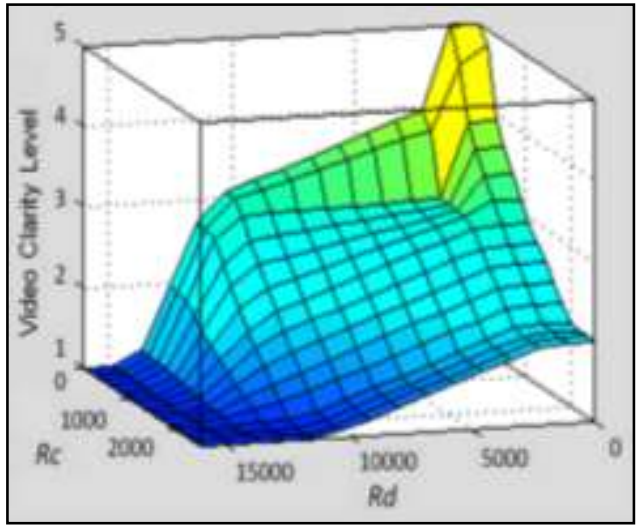

Figure 10. Rule Surface MATLAB Toolbox on Default Mode

\subsection{Performance test on default mode}

the purpose of the trial is to evaluate the performance of fis algorithm especially in the default mode. the fis algorithm as real-time vqa works well if the fis output visual perception close to subjective perception of the tv user. the trial process is performed on 5 video clarity conditions according to user's perception. each condition are 100 times tested. figure 11 shows the results of trials in this session. the average accuracy percentage on the real-time vqa is parameter that observed in this trial. accuracy percentage is the percentage of conformity between real-time VQA perception and user's perception.

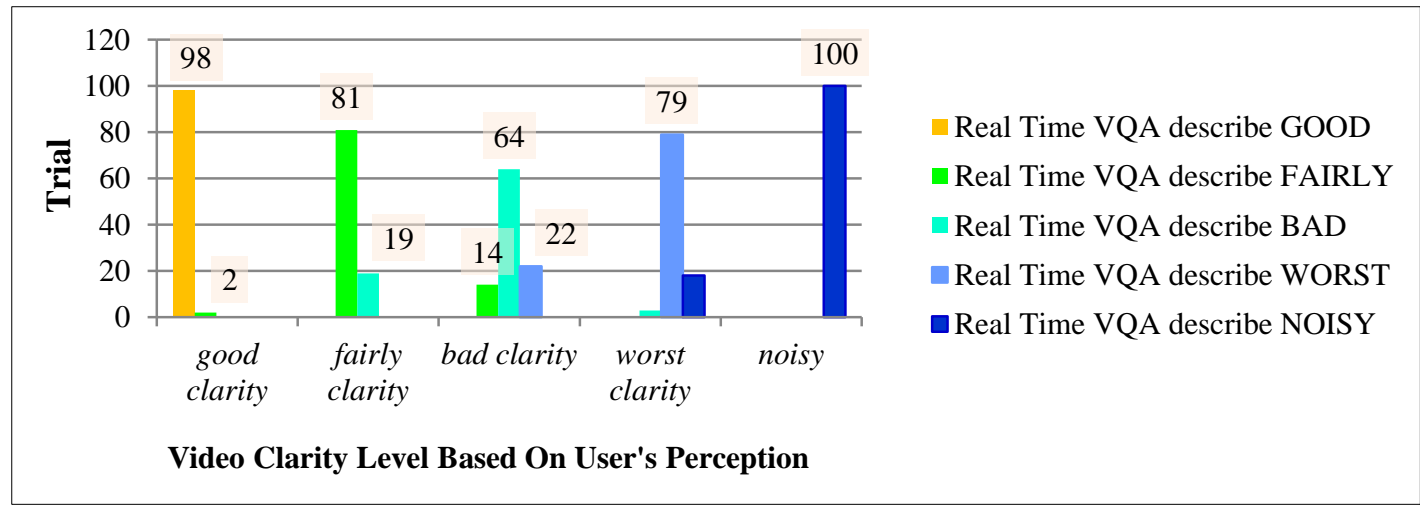

Figure 11. Real-time VQA Performance Test on Default Mode

Test results show some important points, among others are as follows:

a. VQA has good performance when the video clarity level is in good clarity condition. From 100 trials, 98 times real-time VQA declare good clarity and the rest declare fairly clarity. In other words, the accuracy percentage reached $98 \%$.

b. VQA has good performance when the video clarity level is in a fairly clarity condition that reaches $81 \%$, the worst clarity condition reaches $79 \%$, and the noisy condition reaches $100 \%$. 
c. VQA has poor performance when the video clarity level is in bad clarity condition. The accuracy percentage only reaches $64 \%$. This percentage is the lowest performance of real-time VQA.

\subsection{Performance Test on User Mode}

This trial conducted by involving the user. Randomly, a user is selected to assess the video clarity level on the TV screen. User's assessment conducted by pressing the button on the prototype PCB. The result of assessment by user, automatically changes fuzzy membership functions on the microcontroller (discussion on sub-chapter 3.3.2). Figure 12 shows the results of the real-time VQA performance test on the 1st user.

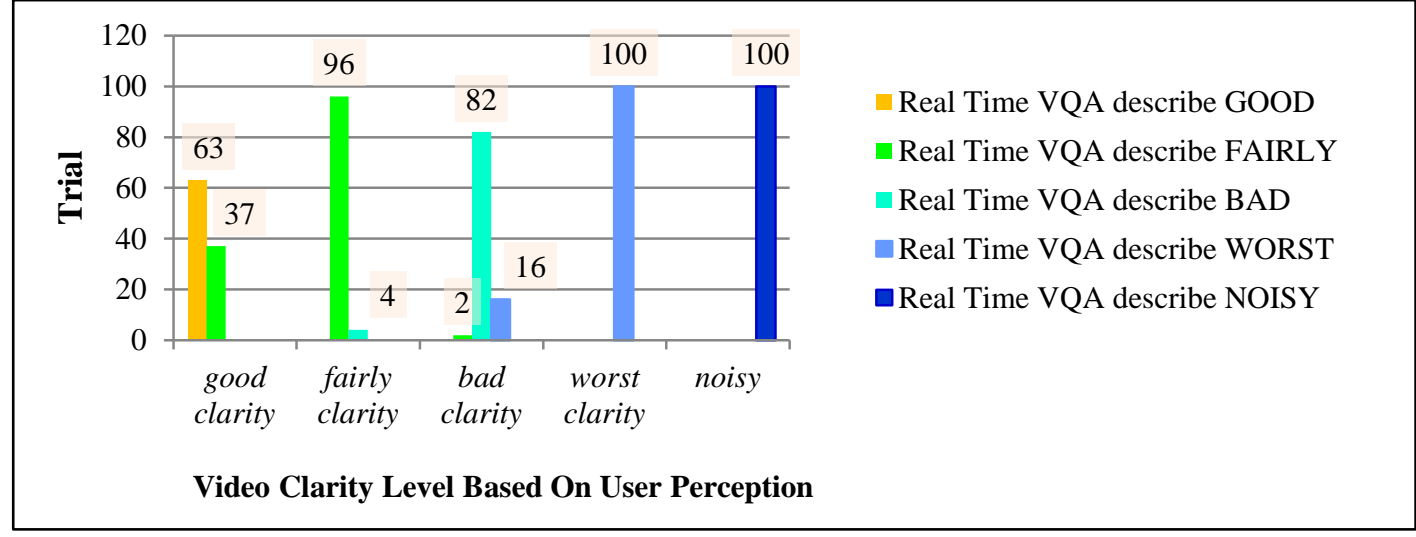

Figure 12. Real-time VQA Performance Test on $1^{\text {st }}$ User (User Mode)

Test results on the Figure 12, show some important points, among others are as follows:

a. VQA has good performance when the video clarity level is in fairly clarity condition. Accuracy percentage reached $96 \%$. Accuracy percentage on the bad clarity condition reaches $82 \%$, accuracy percentage on the worst clarity condition reaches $100 \%$, and accuracy percentage on the noisy condition reaches $100 \%$.

b. VQA has poor performance when the video clarity level is in good clarity condition the accuracy percentage is only $63 \%$. This percentage is the lowest performance of real-time VQA especially in this session.

While Figure 13 shows the membership function setting obtained from user's assessment via button. Figure 13 shows how the user's assessment could change triangle form on fuzzy membership function. It can be compare with Figure 9.

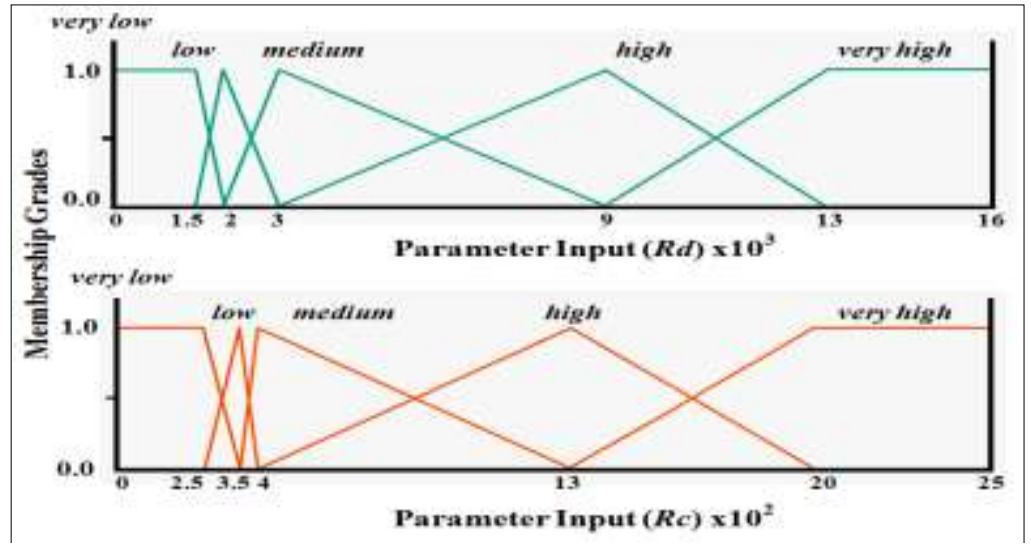

Figure 13. Fuzzy Membership Function on $1^{\text {st }}$ User's assessment 


\subsection{Overall performance test}

This trials process only repeats of trial that has been conducted on the sub-chapter 4.3. But in this sub-chapter, trial process conducted on different users. Table 3 shows the results of the implementation trials. The results of trials in sub-chapters 4.2 and 4.3 are also included in the Table 3.

Table 3. Real-time VQA Performance Test on Different User

\begin{tabular}{|c|c|c|c|c|c|c|}
\hline \multirow[b]{2}{*}{ Performance Trial } & \multicolumn{5}{|c|}{$\begin{array}{c}\text { Accuracy Percentage on Various Video Clarity Level } \\
\text { Condition (\%) }\end{array}$} & \multirow[b]{2}{*}{$\begin{array}{c}\text { Average Accuracy } \\
\text { Percentage (\%) }\end{array}$} \\
\hline & $\begin{array}{l}\text { Good } \\
\text { Clarity }\end{array}$ & $\begin{array}{l}\text { Fairly } \\
\text { Clarity }\end{array}$ & $\begin{array}{l}\text { Bad } \\
\text { Clarity }\end{array}$ & $\begin{array}{l}\text { Worst } \\
\text { Clarity }\end{array}$ & Noisy & \\
\hline Default Mode & 98 & 81 & 64 & 79 & 100 & 80.6 \\
\hline $1^{\text {st }}$ User & 63 & 96 & 82 & 100 & 100 & 88.2 \\
\hline $2^{\text {nd }}$ User & 89 & 84 & 48 & 74 & 100 & 79 \\
\hline $3^{\text {hd }}$ User & 80 & 81 & 65 & 98 & 100 & 84.8 \\
\hline $4^{\text {th }}$ User & 83 & 91 & 69 & 87 & 100 & 86 \\
\hline $5^{\text {th }}$ User & 89 & 96 & 44 & 93 & 100 & 84.4 \\
\hline $6^{\text {th }}$ User & 49 & 86 & 95 & 100 & 100 & 86 \\
\hline $7^{\text {th }}$ User & 90 & 84 & 53 & 100 & 100 & 85.4 \\
\hline $8^{\text {th }}$ User & 87 & 89 & 49 & 96 & 100 & 84.2 \\
\hline $9^{\text {th }}$ User & 73 & 76 & 92 & 93 & 100 & 86.8 \\
\hline $10^{\text {th }}$ User & 72 & 96 & 66 & 82 & 100 & 83.2 \\
\hline $11^{\text {th }}$ User & 59 & 71 & 93 & 100 & 100 & 84.6 \\
\hline $12^{\text {th }}$ User & 73 & 79 & 54 & 80 & 100 & 77.2 \\
\hline $13^{\text {th }}$ User & 46 & 72 & 77 & 94 & 100 & 77.8 \\
\hline $14^{\text {th }}$ User & 87 & 84 & 51 & 93 & 100 & 83 \\
\hline $15^{\text {th }}$ User & 76 & 86 & 48 & 88 & 100 & 79.6 \\
\hline Lowest Accura & Percenta & & & & & \\
\hline
\end{tabular}

Test results show some important points, among others are as follows:

a. In general, the implementation of FIS algorithm on real-time VQA shows good performance. This shown from the average percentage of the lowest accuracy that reach $77.2 \%$ and the highest reach $88.2 \%$.

b. From trials that performed on 1st user to the 15th user, it can be concluded that they has same visual perception in assessing the video clarity level only in the noisy condition. This is evidenced from the accuracy percentage ranging from user to 1 to 15 users. The accuracy percentage under noisy conditions is $100 \%$. While in other video clarity level conditions, they have different visual perceptions.

\subsection{Overall Results Analysis}

The analysis of overall trials in this study is obtained from the results in Table 3. Important points, among others are as follows:

a. Overall, real-time VQA performance still needs to be improved especially when video clarity is in good clarity condition. Good clarity condition is the ultimate goal sought for each analog TV user, so setting membership function on fuzzy algorithm especially when good clarity condition still need special attention.

b. Besides that, it still needs to be improved when video clarity levels on bad clarity condition. When referring to trial result on chapter 4.1 , the Rd parameter range when the video clarity level in the bad clarity conditions is the widest between 1,657 and 11,590. Thus, the degree of obscurity in the fuzzy membership function also needs special attention. Assessment result on this condition shows that 5 membership function triangles that established are not enough for real-time VQA to describe the video clarity level.

\section{Conclusion}

This study is an effort to build tracking antenna system for analog TV especially concern on real-time VQA. It is not easy to build real-time VQA that corresponding to subjectivity and diversity of user's visual perceptions. Adaptive FIS algorithm with membership function tuning was used as real-time VQA. In this case, the membership function of $R d$ and $R c$ parameters can be changed according to user's perception as simply only by pressing button. 
The results show that the real-time VQA have good performance. This shown from the average accuracy percentage of lowest that reaches $77.2 \%$ and highest that reaches $88.2 \%$. Even so, real-time VQA performance needs to be improved, especially when the video clarity condition is on good clarity and bad clarity. This improvement problem will be future study.

\section{Acknowledgment}

Acknowledgment of the authors to Direktorat Riset dan Pengabdian Masyarakat (DRPM), Direktorat Jenderal Penguatan Riset dan Pengembangan, Kementerian Riset, Teknologi, dan Pendidikan Tinggi Republik Indonesia (KEMENRISTEK DIKTI) for the support of Research Funding Year 2017 on research scheme Penelitian Kerjasama Antar Perguruan Tinggi (PEKERTI), that conducted by Universitas Bhayangkara Surabaya (UBHARA) and Institut Teknologi Sepuluh Nopember (ITS) Surabaya, with the contract number 010/SP2H/K2/KM/2017, on May 4, 2017. Acknowledgment also to ITS Robotics, Information and Communication Technology Center, ITS Telecommunication Network Laboratory and UBHARA Surabaya Electrical Engineering Laboratory.

\section{References}

[1] Miawarni H, Setijadi E. Antenna Tracking System Based on Pulse of Synchronization CVBS: Design System And Analyze. International Electronics Symposium (IES). Bali. 2016; 18: 228.

[2] Miawarni H, Hidayat MM, Sumpeno S, Setijadi E. Tracking System for Indoor TV Antenna Based on CVBS Signal Processing. Jurnal Elektronika dan Telekomunikasi (JET). Surabaya. 2017; 17(2): 48-55.

[3] Suardinata, Kamalrulnuzam. A Fuzzy Logic Classification of Incoming Packet for Voip. TELKOMNIKA (Telecommunication, Computing, Electronics and Control). 2010; 8(2): 165-174.

[4] Bock F, Steudel A, Clausert H, Glesner M. A Fuzzy Rule-Based System for the Subjective Quality Evaluation ff Distorted Images. Sixth International Conference on Image Processing and its Applications. Dublin. 1997; 1: 448.

[5] Yao S, Lin W, Lu Z, Ong E, Etoh M. Objective Quality Assessment for Compressed Video. IEEE Proceedings of the International Symposium on Circuits and Systems (ISCAS). Bangkok. 2003; 4: 897.

[6] Alreshoodi M, Danish E, Woods J, Fernando A, Alwis C. Prediction of Perceptual Quality for Mobile Video Using Fuzzy Inference Systems. IEEE Transactions on Consumer Electronics. 2015; 61(4): 569-554.

[7] Pitas C, Charilas D, Panagopoulos A, Constantinou P. Adaptive Neuro-Fuzzy Inference Models for Speech and Video Quality Prediction in Real-World Mobile Communication Networks. IEEE Wireless Communications. 2013; 20(3): 80-88.

[8] Danish E, Fernando A, Alreshoodi M, Woods J. A Hybrid Prediction Model for Video Quality by QOS/QoE Mapping in Wireless Streaming. IEEE International Conference on Communication Workshop (ICCW). London. 2015; 7: 1723.

[9] Alreshoodi M, Olufemi A, Woods J, Walker S. Fuzzy Logic Inference System-Based Hybrid Quality Prediction Model for Wireless 4K UHD H.265-Coded Video Streaming. IET Networks. 2015; 4(6): 296-303.

[10] Winkler S. Video Quality and Beyond. Proceeding on European Signal Processing Conference. Poznan. 2007; 15: 150

[11] Winkler S, Mohandas P. The Evolution of Video Quality Measurement: From PSNR to Hybrid Metrics. IEEE Transactions on Broadcasting. 2008; 54(3): 660-668.

[12] Rekha KB, Kumar R. High Quality Video Assessment Using Salient Features. Indonesian Journal of Electrical Engineering and Computer Science (IJEECS). 2017; 7(3): 767-772.

[13] Shakir M, Rehman O, Abbas Z, Masood A, Shahid W. Evaluation of Video Quality in Wireless Multimedia Sensor Networks. International Journal of Electrical and Computer Engineering (IJECE). 2016; 6(1): 223-234.

[14] Yanyu W, Shirin M. Video Compression Encoding and Decoding Mechanism of H.264 Based on FPGA. TELKOMNIKA (Telecommunication, Computing, Electronics and Control). 2016; 14(2): 85-91.

[15] White T, Reid G. Quality of PAL Colour TV Pictures Impaired by Random Noise: Stability of Subjective Assessment. IEE Proceedings on Communications, Radar and Signal Processing. 1981; 128: 231.

[16] International Telecommunication Union. Recommendation ITU-T P.800.1. Mean Opinion Score (MOS) Terminology. Geneva: ITU; 2006.

[17] Fischer W. Digital Video and Audio Broadcasting Technology: A Practical Engineering Guide. Berlin: Springer. 2010.

[18] Ross TJ. Fuzzy Logic with Engineering Applications. West Sussex. Wiley. 2010. 